\title{
Estimating racial health disparities among adverse birth outcomes as deviations from the population rates
}

\author{
James A. Thompson ${ }^{1 *}$ (D) and Melissa A. Suter ${ }^{2}$
}

\begin{abstract}
Background: Despite significant research, the reasons for racial health disparities among adverse birth outcomes $(A B O)$ remain largely unknown. The bulk of research into racial health disparities among ABO in the United States has concentrated on the risk of race and ethnic groups relative to the specific sub-population of non-Hispanic white women and their children. The objective of this study was to estimate the racial and ethnic risks among a set of neonatal and maternal health disparities while minimizing bias attributable to how the baseline risk was established.

Methods: All birth records were obtained from the United States Natality database for the years 2014 to 2017. A Bayesian modeling approach was used to estimate the risk disparity for disorders by race. The estimation of the racespecific risks used a sum-to-zero constraint for the race regression coefficients.

Results: Estimating racial health disparities relative to the overall population rate yielded novel results and identified perinatal health disparities for all the race groups studied.

Conclusions: Unbiased risk estimates for racial disparities among $A B O$ are now available for stimulating and initiating more complex causal modeling that can lead to understanding how racial health disparities for $A B O$ are mediated and how they can be prevented.
\end{abstract}

Keywords: Bayesian, Sum-to-zero, Maternal, Neonatal, Racial health disparity, Adverse birth outcomes

\section{Background}

In 2017, the United States infant mortality rate (IMR) was 5.8 deaths per 1000 live infant births and ranked an abysmal number 33 out of the 36 countries evaluated [1]. Even more concerning than the overall IMR is that nonHispanic black infants had 2.4 times the rate of infant mortality compared to non-Hispanic white infants [2]. The IMR provides a valuable summary for both maternal and child adverse birth outcomes (ABO) and is often used to evaluate both national health care systems and racial health disparities $[3,4]$. In the United States, infant

\footnotetext{
* Correspondence: jthompson@cvm.tamu.edu

${ }^{1}$ College of Veterinary Medicine and Biomedical Science, Texas A\&M University, College Station, TX 77843-4475, USA

Full list of author information is available at the end of the article
}

mortality is commonly grouped into three common causes: birth defects, preterm birth and maternal pregnancy complications. Maternal pregnancy conditions include both severe maternal morbidities (SMM) that occur with labor and delivery and metabolic disorders like gestational diabetes, gestational hypertension and hypertension eclampsia [5]. Each of these common causes of $\mathrm{ABO}$ is affected by profound racial health disparities $[2,6]$.

Despite significant research, the reasons for these racial health disparities remain largely unknown. In their strategic planning, the National Institute on Minority Health and Health Disparities (NIMHD) has long defined a health disparity as a group's deviation in risk from the overall population risk [7], emphasizing that pair-wise comparisons between two specific sub-populations do not allow

(c) The Author(s). 2020 Open Access This article is licensed under a Creative Commons Attribution 4.0 International License, which permits use, sharing, adaptation, distribution and reproduction in any medium or format, as long as you give appropriate credit to the original author(s) and the source, provide a link to the Creative Commons licence, and indicate if changes were made. The images or other third party material in this article are included in the article's Creative Commons licence, unless indicated otherwise in a credit line to the material. If material is not included in the article's Creative Commons licence and your intended use is not permitted by statutory regulation or exceeds the permitted use, you will need to obtain permission directly from the copyright holder. To view a copy of this licence, visit http://creativecommons.org/licenses/by/4.0/ The Creative Commons Public Domain Dedication waiver (http://creativecommons.org/publicdomain/zero/1.0/) applies to the data made available in this article, unless otherwise stated in a credit line to the data. 
for assessment of the overall degree of disparity in an entire population with more than two groups $[8,9]$. However the bulk of research into racial health disparities among $\mathrm{ABO}$ has concentrated on the risk of race and ethnic groups relative to the specific sub-population of nonHispanic white women and their children. The objective of this study was to estimate the racial risks among a set of neonatal and maternal health disparities while minimizing bias attributable to how the baseline risk was established. Once racial health disparities are objectively defined and accurately estimated, more specific causal modeling can be addressed. In future research, extending the model that estimated these risks will be able to identify race-specific mediators of race-specific health risks.

\section{Methods}

All birth records were obtained from the United States Natality database for the years 2014 to 2017 [10]. The variables extracted from the database included maternal race and ethnicity, maternal pregnancy conditions, severe maternal morbidities, neonatal conditions, neonatal treatments, birth defects and neonatal death. Maternal race and ethnicity was retained as eight categories: nonHispanic white, non-Hispanic black, American Indian and Alaska Native (AIAN), Asian, Native Hawaiian and Other Pacific Islanders (NHOPI), Hispanic, mixed and unknown. All eight categories were used in the analysis and referred to as "race." Estimates for maternal races recorded as "mixed" and "unknown" are not reported. Infants were assigned the maternal race for all births regardless of the paternal race. Maternal pregnancy conditions included gestational diabetes, gestational hypertension and hypertension eclampsia and severe maternal morbidities included induction of labor, caesarian section, maternal transfusion, perineal laceration, ruptured uterus, unplanned hysterectomy and admission to intensive care unit (ICU). Neonatal outcomes included birthweight, gestation length, Apgar score and the occurrence of seizures. Neonatal treatments included antibiotics, administration of surfactant, whether ventilation was started, whether ventilation was prolonged $(>6 \mathrm{~h})$ and whether the child was admitted to a neonatal intensive care (NICU). Very preterm delivery (VPTD) was defined as a gestation period of less than 32 weeks. Preterm delivery (PTD) was defined as gestation period of less than 37 weeks. Large for gestational age (LGA) was defined as the largest $10 \%$ of birth weights by gestation length and small for gestational age (SGA) as the smallest $10 \%$ of birthweight by gestation length. Birth defects included anencephaly, meningomyelocele/spina bifida, cyanotic congenital heart disease, diaphragmatic hernia, omphalocele, gastroschisis, limb reduction defect, cleft lip (with or without cleft palate), cleft palate alone, hypospadias, Down syndrome and chromosomal disorders. Neonatal death was defined as a death that occurred before the birth certificate was completed. Inclusion criteria are singleton births with all variables of interest available. Of the 15,261,278 singleton births available in the database, 214,036 were excluded due to missing variables, leaving 15,047,242 births to be used in the analysis.

A Bayesian modeling approach was used to estimate the odds ratios for disorders by race. The estimation of the race-specific risks used a sum-to-zero constraint for the adjusted race regression coefficients as follows: Case counts were cross tabulated by $i=8$ race/ethnicities and $j=34$ disorders. For each row in the table, $Y_{i j}$ was the count of cases at birth and $n_{i}$ the count of births. The counts $\left(Y_{i j}\right)$ were modeled as independent Binomial distributions conditional on an unknown rate parameter $\left(u_{i j}\right)$.

$$
Y_{i j} \sim \operatorname{Binomial}\left(u_{i j}, n_{i}\right)
$$

The logit of the rate parameter was then provided a vague Normal prior with a mean $=0$ and variance $=1000$.

$$
\operatorname{Logit}\left(u_{i j}\right) \sim N(0,1000)
$$

An adjusted rate parameter $\left(u^{\prime}{ }_{i j}\right)$ was estimated constrained to sum to zero by subtracting the race means ( $\bar{u}_{i}$ ) from the unconstrained rate parameter at each iteration of the Markov Chain.

$$
u_{i j}^{\prime}=u_{i j}-\bar{u}_{i}
$$

The odds ratio was estimated as the inverse log of the adjusted rate parameter.

$$
O R_{i j}=e^{u^{\prime} i j}
$$

For the estimate of percent risk increase, the race risk was estimated by the rate parameter $\left(u_{i j}\right)$ and the expected rate was estimated from the race mean parameter $\left(\bar{u}_{i}\right) . P$-values were defined as the posterior probability of the percent increase being greater than zero and was taken directly from the full posterior distribution [11]. The implementation used Markov Chain Monte Carlo (MCMC) and the software OpenBUGS 3.2.3 [12]. One thousand iterations were allowed for burn-in and the next 10,000 iterations were collected for the posterior distribution. Monitoring chains for the adjusted rate parameter and the race means was used to determine convergence. The code for analyses and data are available in additional files.

\section{Results}

For all results, the percent increase in risk, Bayesian credibility intervals and $p$-values are presented in Table 1.

White non-Hispanic women had increased risk for developing gestational hypertension, having a caesarian 


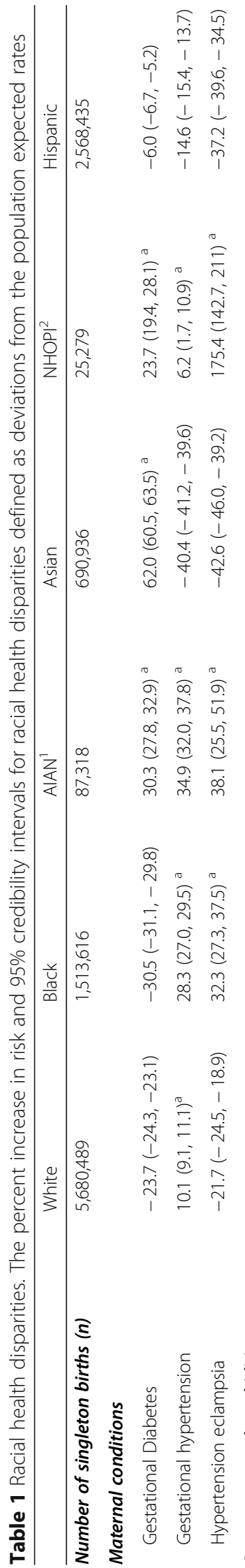

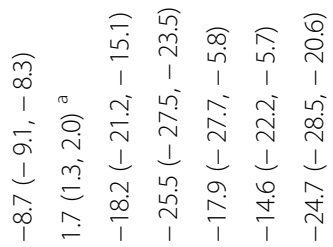

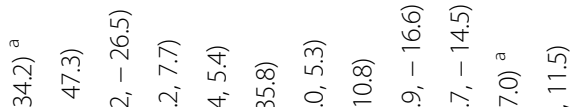

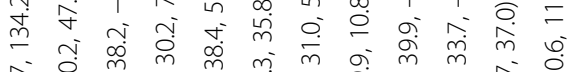

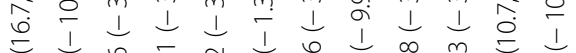

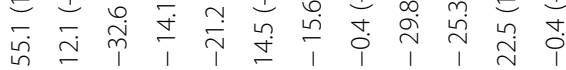

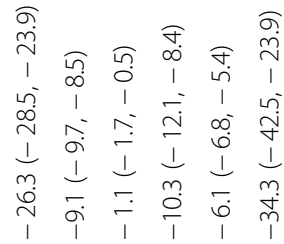

$\underset{6}{6}$

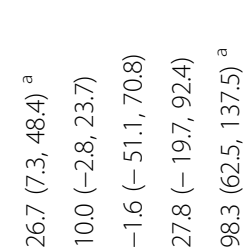

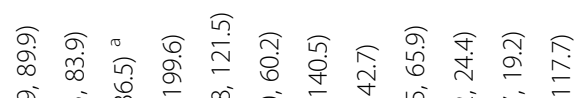

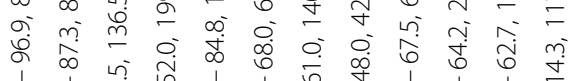

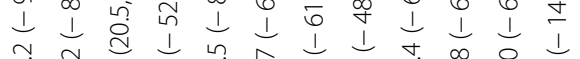

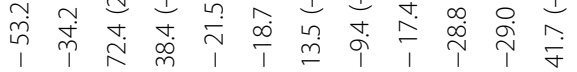

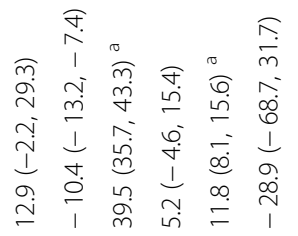

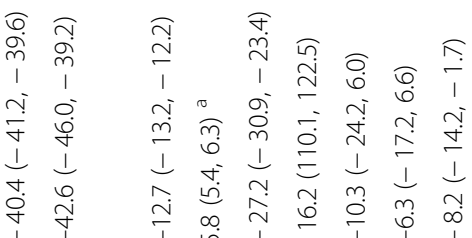

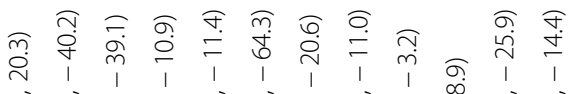

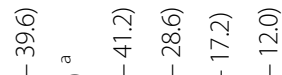

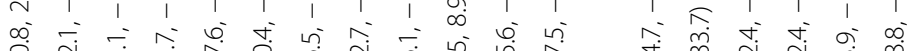

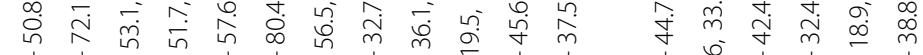
$\begin{array}{llllllllllllll}1 & 1 & 1 & 1 & 1 & 1 & 1 & 1 & 1 & 1 & 1 & 1\end{array}$

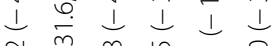

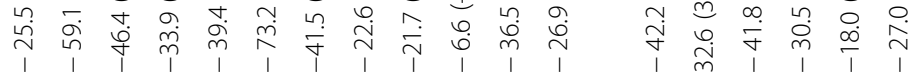

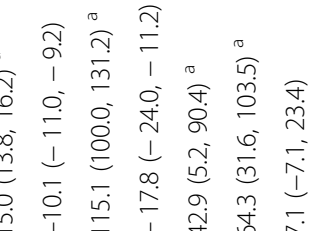

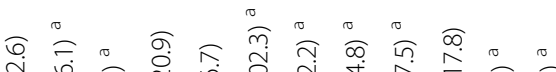

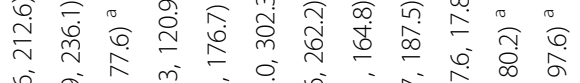

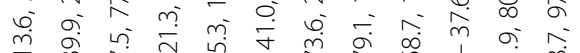

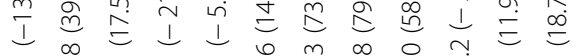

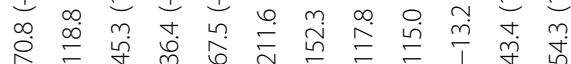

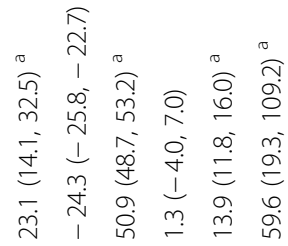

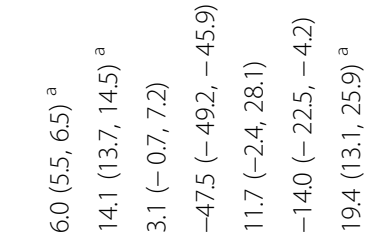

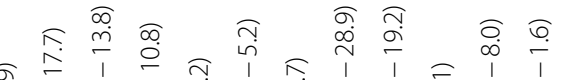

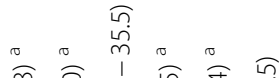

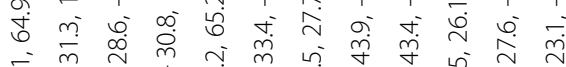

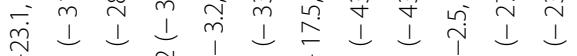

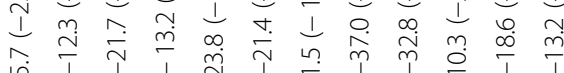
m ก.

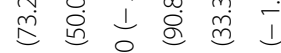

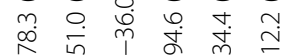

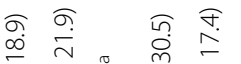

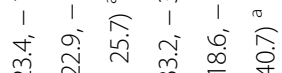

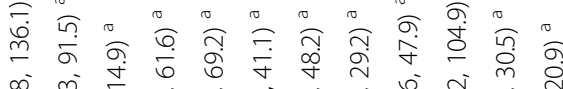

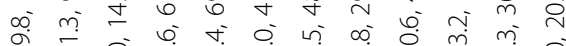

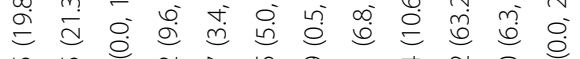

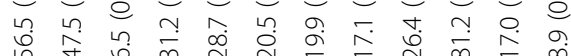

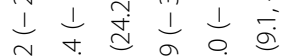

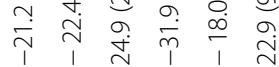

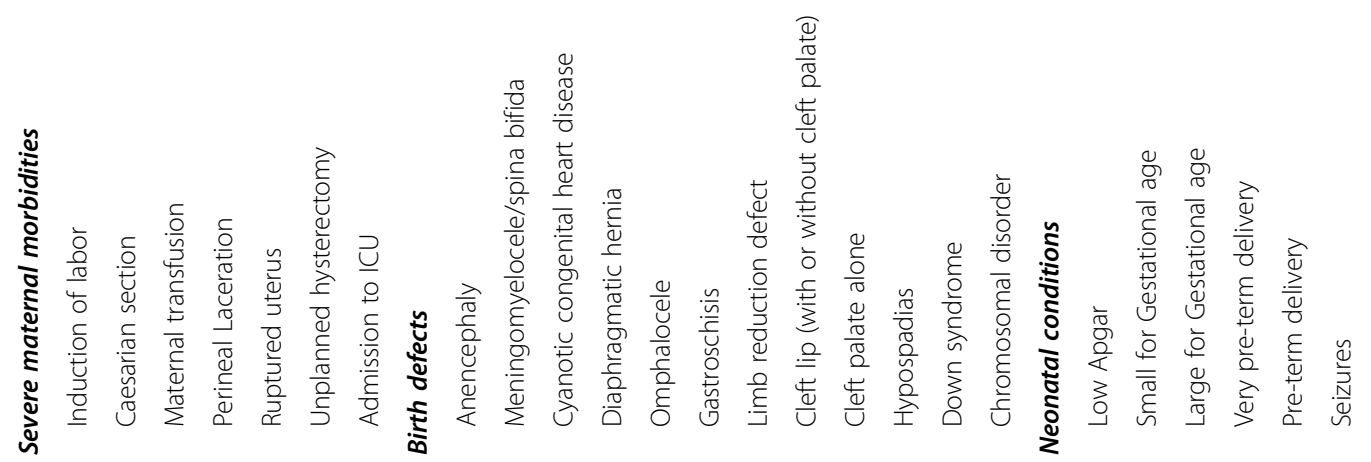




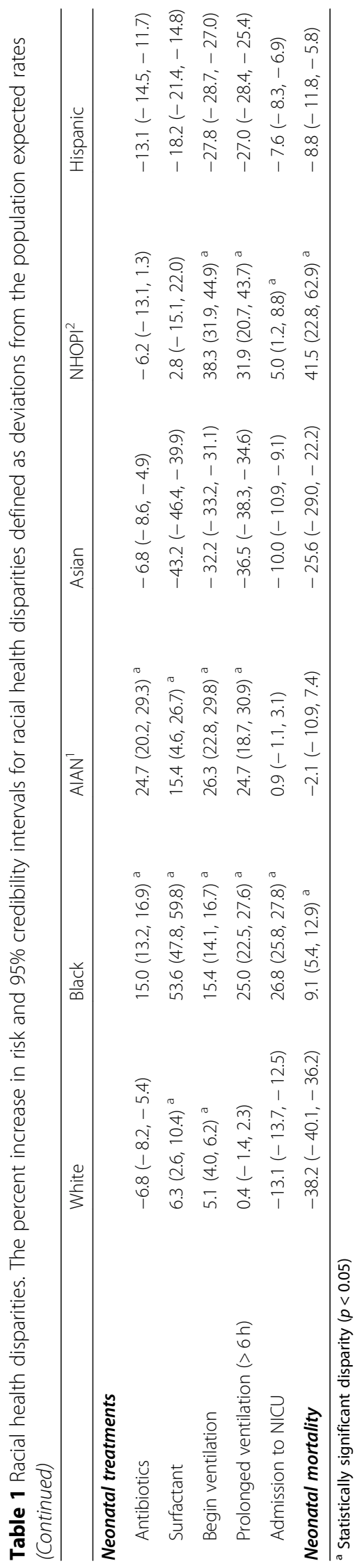


section and experiencing a perineal laceration. Children of these women had increased risk for LGA, having seizures and had increased risk for 10 monitored birth defects and both Down syndrome and chromosomal disorders.

Women who were classified as black and nonHispanic were more likely to develop gestational hypertension and hypertension eclampsia. Increased risks for SMM included induction of labor, caesarian section and admission to the ICU. Their children had increased risk for VPTD, PTD and SGA as well as low Apgar scores. They were more likely to be treated with antibiotics and surfactant and more likely to be started on ventilation, to have received prolonged ventilation and more likely to have been admitted to the NICU.

American Indian and Alaska Native (AIAN) women had increased risk for developing gestational diabetes, gestational hypertension and hypertension eclampsia. Increased risk for SMM included induction of labor, maternal transfusion and admission to ICU. Neonatal risks were increased for LGA, PTD, low Apgar and seizures. These children had increased risk for birth defects, including meningomyelocele/spina bifida, cyanotic congenital heart disease, gastroschisis, limb reduction defect, cleft lip, cleft palate as well as for Down syndrome and chromosomal disorders. Increased risks for neonatal treatments included treatment with antibiotics, surfactant, beginning ventilation, prolonged ventilation and admission to NICU.

Native Hawaiian and Other Pacific Islanders (NHOPI) women had increased risk of developing gestational diabetes, hypertension and hypertension eclampsia. Increased risks for SMM included for maternal transfusion and admission to ICU. Children of NHOPI women had increased risk of PTD and LGA and the group of birth defects referred to as cyanotic congenital heart disease. These children were more likely to have been started on ventilation, received prolonged ventilation and to have been admitted to NICU.

Asian and Hispanic women were relatively free of ABO. The exceptions included that Asian women had increased risk of developing gestational diabetes and had increased risk for caesarian section or perineal laceration. Asian children had no increased risks for the ABO studied. Hispanic women had a very small elevation of risk for caesarian section and their children had increased risk for Down syndrome and anencephaly.

\section{Discussion}

For the purpose of the current study, the authors define "disparity" as any increase in risk for $\mathrm{ABO}$ based on an individual's race or ethnicity when compared to the overall population risk rate. The NIMHD cites a colorful debate regarding the use of the word "disparity" but clarify, for the sake of strategic planning, that racial health disparity means any increase regardless of whether the difference seems unjust with "unjust" meaning attributable to a policy that affects health inequitably among races [7]. This approach is most appropriate because causal modeling must be relatively well defined before a disease cause can be debated as "just or unjust." For example, the Bayesian patient-based model of the current study could be expanded to include hierarchal variables that could demonstrate risks of social policies that would satisfy most criteria for an "unjust" cause. The NIMHD in providing this working definition is stipulating that the risks should be estimated for each group relative to the overall population risk. The objective of the current study was to estimate racial disparity risk for $34 \mathrm{ABOs}$ among 15 million births in the United States over a recent four-year period. In spite of the NIMHD working definition for disparity, there is a large body of literature describing racial health disparities comparing the disparity to the specific subpopulation of non-Hispanic white women and their children. This is true for pregnancy conditions and SMM [5], birth defects [13-15] and neonatal morbidities [2, 16, 17]. The current modeling was performed using a Bayesian approach and a readily available software package and by using a sum-to-zero constraint for the model coefficients [18]. This MCMC implementation maintains the favorable Bayesian properties of full posterior distributions for all of the parameters estimated. Point estimates of the pairwise comparisons can be derived from results of the current study from the ratio of the median of the two odds ratios. Furthermore, with the data and model available in the additional files, the full posterior distribution of any pairwise comparison can be estimated.

Much of the existing literature that describes racial disparities adjusts the disparity risk estimate for covariates like socioeconomic status (SES), for example [2, 5, 9, 13-17]. The rationale for conditioning on covariates has not always been clear. Following a unifying and serviceable definition for confounding [19], there are no backdoor paths between race and any of the outcomes in the current study because none of the factors can "cause" race. Backdoor paths for the more abstract construct "racism" can be envisioned and confounding control defended in those models [4] but controlling for confounding by racism was not an objective of the study and should not be performed for descriptions of potential racial disparities. For the unchangeable factor of race, any covariates used for conditioning should be considered potential mediators and it is the effect of the mediator in changing the risk estimate for each race that is of interest, not just the resultant conditional risk [20]. Future work should evaluate specific mediation models and effects but should start with the best possible estimates for the risk of racial health disparity. 
The current study yielded novel risk estimates for white non-Hispanic women and their children. These racial health disparities should be considered potentially comorbid conditions. Among non-Hispanic women and their children, Down syndrome is known to be associated with comorbidities including birth defects, seizures and multiple other conditions that can lead neonatal death [21]. In another causal web, LGA, gestational hypertension, and either caesarian section or perineal laceration are known to be associated with both gestational weight gain and pre-pregnancy Body Mass Index [22]. While induction of labor and caesarian deliveries are not considered severe compared to other maternal morbidities studied, they have been included as a maternal morbidity. However, induction of labor or caesarian section likely contributes to avoidance of less favorable maternal and neonatal outcomes and because they often increase the number of good outcomes, they should not be automatically classified as SMM. A limitation of this study is that the database does not identify indication for cesarean delivery. Furthermore, information on whether PTD was indicated or spontaneous is also not available. Further research on how these associate with health disparities is needed. It is possible that the racial disparities among caesarian delivery and labor induction may be explained by comorbid conditions.

Changing the baseline rate to the population rate rather than the rate among non-Hispanic white women and their children lowered the estimated risk rate for many of the ABO for non-Hispanic black women and their children. This results because white non-Hispanic women and their children have lower than population average risk for multiple ABO. This was especially notable among VPTD, PTD and SGA and a well-known wide range of comorbidities, including neonatal mortality. In spite of the lowered risk estimates, the list of comorbidities remains relatively unchanged and included pregnancy complications, SMM, neonatal morbidity and neonatal treatments. These disparities were both statistically and clinically significant and the need further investigation into the causes continues to be urgent.

The current results estimate a wide range of comorbidities for AIAN women and their children. Included among the comorbidities are five birth defects for which the risk for children of AIAN women are at least double the risk rate of the overall population. These birth defects include meningomyelocele/spina bifida; gastroschisis; limb reduction defect; cleft lip and cleft palate.

Women classified as NHOPI had increased risk of pregnancy complications including a more than doubling of risk for hypertension eclampsia and a near doubling of risk for admission to ICU. These two conditions are considered to be causally linked [23] and are jointly caused by maternal obesity, which is more prevalent among NHOPI women [24]. The children of NHOPI women had increased risk of cyanotic congenital heart disease, LGA and PTD and to be treated with short- and long-term ventilation and admission to NICU. In addition to causing maternal conditions, maternal obesity is also associated with both LGA and PTD [21]. This cluster of comorbidity may also include a causal connection between maternal obesity and cyanotic congenital heart disease [2527] and the treatment sequelae [28]. Racial health disparities for birth defects among children of NHOPI women have been reported but the risks were estimated relative to non-Hispanic white women and their children and the risk was adjusted for maternal age without a reporting of the mediation effect of maternal age $[13,14]$. More study is needed to evaluate the large cluster of potentially causal associations.

The authors of a recent special journal edition claimed that what were needed most following a refocusing definition were analytic methods addressing the ability to draw causal inferences from observational studies [8, 29]. We anticipate that the ultimate causal explanations will build on the concept that multiple disorders within a racial, ethnic or otherwise defined disadvantaged group are comorbidities and share common causal elements. All of these causal elements will be downstream from race and thus should be considered potential mediators of the effects of racial health disparity but not confounders. How the comorbidities fit into a causal web as mediators will present a vexing problem. The list of comorbidities is relatively well defined, however, the causal ordering of common causal factors among the many pregnancy complications, SMM, neonatal morbidity and neonatal treatments will always be debatable. However, causal modeling is encouraged and reports should include the causal modeling assumptions which will enable the long-term goal of identifying causes of racial disparities that can be addressed.

\section{Conclusions}

Estimating racial health disparities relative to the overall population expected rate yielded novel results and identified perinatal health disparities for all the race and ethnic groups studied. Unbiased risk estimates for racial and ethnic disparities among ABO should be used to initiate more complex causal modeling that can lead to understanding how racial health disparities for $\mathrm{ABO}$ are mediated and how they can be prevented.

\section{Supplementary information}

Supplementary information accompanies this paper at https://doi.org/10. 1186/s12884-020-2847-9.

Additional file 1. WinBUGS code and data. This code and these data can be used to repeat the analyses reported here. 


\section{Abbreviations}

AIAN: American Indian and Alaska Native; ICU: Intensive care unit; IRB: Institutional review board; LGA: Large for gestational age; MCMC: Markov Chain Monte Carlo; NHOPI: Native Hawaiian and other Pacific Islanders; NICU: Neonatal intensive care unit; PTD: Preterm delivery; SES: Socioeconomic status; SGA: Small for gestational age; SMM: Severe maternal morbidity; VPTD: Very preterm delivery

\section{Acknowledgements}

Not applicable

\section{Authors' contributions}

Both authors contributed to the study's conception. JAT performed the analyses. MAT lead the development of discussion. Both authors prepared and approved the manuscript.

\section{Funding}

This research was funded in part by a grant from the National Institutes of Health: R03CA208021. The National Institutes of Health had no role in the design of the study, collection, analysis, interpretation of data, and in writing the manuscript.

\section{Availability of data and materials}

The database used in the study is publically available (https://www.cdc.gov/ nchs/data_access/vitalstatsonline.htm). These data collected by the National Center for Health Statistics may be used only for the purpose of health statistical reporting and analysis. The authors consented a data use agreement (https://www.cdc.gov/nchs/data_access/restrictions.htm).

The tabulated data and the analysis code for OpenBUGS are available in Additional file 1

\section{Ethics approval and consent to participate}

This study was evaluated by the Texas A\&M Institutional Review Board (IRB) and determined to be exempt from IRB review.

\section{Consent for publication}

Not applicable.

\section{Competing interests}

The authors declare that they have no competing interests.

\section{Author details}

${ }^{1}$ College of Veterinary Medicine and Biomedical Science, Texas A\&M University, College Station, TX 77843-4475, USA. ²Department of Obstetrics and Gynecology, Baylor College of Medicine, 1 Baylor Plaza, Houston, TX 77030, USA.

\section{Received: 10 December 2019 Accepted: 27 February 2020} Published online: 12 March 2020

\section{References}

1. OECD. Infant Mortality Rates 2019. 08/13/2019 Available from: https://data. oecd.org/healthstat/infant-mortality-rates.htm.

2. MacDorman MF. Race and ethnic disparities in fetal mortality, preterm birth, and infant mortality in the United States: an overview. Semin Perinatol. 2011;35(4):200-8

3. Matoba N, Collins JW. Racial disparity in infant mortality. Semin Perinatol 2017:41(6):354-9..

4. Alhusen JL, Bower KM, Epstein E, Sharps P. Racial discrimination and adverse birth outcomes: an integrative review. J Midwifery Wom Heal. 2016;61(6): 707-20

5. Howell EA. Reducing disparities in severe maternal morbidity and mortality. Clin Obstet Gynecol. 2018;61(2):387-99.

6. CDC. Severe Maternal Morbidity in the United States.2018 https://www.cdc. gov/reproductivehealth/maternalinfanthealth/severematernalmorbidity.html 9/11/2018

7. Thomson GE, Mitchell F, Williams M, National Research Council (U.S.) Committee on the Review and Assessment of the NIH's Strategic Research Plan And Budget to Reduce and Ultimately Eliminate Health Disparities. Examining the health disparities research plan of the National Institutes of
Health : unfinished business. Washington, DC: National Academy Press; 2006. xv. p. 304.

8. Duran DG, Perez-Stable EJ. Novel approaches to advance minority health and health disparities research. Am J Public Health. 2019;109:S8-S10.

9. Rossen LM, Schoendorf KC. Trends in racial and ethnic disparities in infant mortality rates in the United States, 1989-2006. Am J Public Health. 2014; 104(8):1549-56

10. CDC. Vital Statistics Online Data Portal2019 9/3/2019 9/11/2018. Available from: https://www.cdc.gov/nchs/data_access/vitalstatsonline.htm.

11. Zhang JL. Comparative investigation of three Bayesian p values. Comput Stat Data An. 2014:79:277-91.

12. Lunn D, Spiegelhalter D, Thomas A, Best N. The BUGS project: Evolution, critique and future directions. Stat Med. 2009:28(25):3049-67.

13. Canfield MA, Mai CT, Wang Y, O'Halloran A, Marengo LK, Olney RS, et al. The association between race/ethnicity and major birth defects in the United States, 1999-2007. Am J Public Health. 2014;104(9):E14-23.

14. Le MT, Shumate CJ, Hoyt AT, Wilkinson AV, Canfield MA. The prevalence of birth defects among non-Hispanic Asian/Pacific Islanders and American Indians/Alaska Natives in Texas, 1999-2015. Birth Defects Res. 2019;111(18): 1380-8.

15. Marengo LK, Flood TJ, Ethen MK, Kirby RS, Fisher S, Copeland G, et al. Study of selected birth defects among American Indian/Alaska native population: a multi-state population-based retrospective study, 1999-2007. Birth Defects Res. 2018;110(19):1412-8.

16. Manuck TA. Racial and ethnic differences in preterm birth: a complex, multifactorial problem. Semin Perinatol. 2017:41(8):511-8.

17. Schaaf JM, Liem SMS, Mol BWJ, Abu-Hanna A, Ravelli ACJ. Ethnic and racial disparities in the risk of preterm birth: a systematic review and metaanalysis. Am J Perinat. 2013;30(6):433-50.

18. Lunn D, Jackson C, Best N, Thomas A, Spiegelhalter D. The BUGS book : a practical introduction to Bayesian analysis. Boca Raton: CRC Press, Taylor \& Francis Group; 2013. xvii. p. 381.

19. Greenland S, Pearl J, Robins JM. Causal diagrams for epidemiologic research. Epidemiology. 1999;10(1):37-48.

20. VanderWeele TJ, Robinson WR. On the causal interpretation of race in regressions adjusting for confounding and mediating variables. Epidemiology. 2014:25(4):473-84

21. Thompson AM, Thompson JA. An evaluation of whether a gestational weight gain of 5 to $9 \mathrm{~kg}$ for obese women optimizes maternal and neonatal health risks. Bmc Pregnancy Childb. 2019;19(1):126.

22. Martin T, Smith A, Breatnach CR, Kent E, Shanahan I, Boyle M, et al. Infants born with Down syndrome: burden of disease in the early neonatal period. J Pediatr Us. 2018;193:21-6.

23. Hitti J, Sienas L, Walker S, Benedetti TJ, Easterling T. Contribution of hypertension to severe maternal morbidity. Am J Obstet Gynecol. 2018; 219(4):405.e1-405.e7.

24. Ju AC, Heyman MB, Garber AK, Wojcicki JM. Maternal obesity and risk of preterm birth and low Birthweight in Hawaii PRAMS, 2000-2011. Matern Child HIth J. 2018;22(6):893-902.

25. Boyd HA, Basit S, Behrens I, Leirgul E, Bundgaard H, Wohlfahrt J, et al. Association Between Fetal Congenital Heart Defects and Maternal Risk of Hypertensive Disorders of Pregnancy in the Same Pregnancy and Across Pregnancies. Circulation. 2017;136(1):39 +

26. Brite J, Laughon SK, Troendle J, Mills J. Maternal overweight and obesity and risk of congenital heart defects in offspring. Int J Obesity. 2014;38(6): 878-82.

27. Persson M, Razaz N, Bonamy AKE, Villamor E, Cnattingius S. Maternal overweight and obesity and risk of congenital heart defects. J Am Coll Cardiol. 2019:73(1):44-53.

28. Allen KY, Pittsenbarger Z, Roben E. Neonatal Cardiac Emergencies. Clin Pediatr Emerg M. 2018;19(4):340-4.

29. Jeffries N, Zaslavsky AM, Roux AVD, Creswell JW, Palmer RC, Gregorich SE, et al. Methodological approaches to understanding causes of health disparities. Am J Public Health. 2019;109:S28-S3.

\section{Publisher's Note}

Springer Nature remains neutral with regard to jurisdictional claims in published maps and institutional affiliations. 